\title{
Three-dimensional Hilbert fractal acoustic metamaterials: \\ low-frequency and multi-band sound insulation
}

\author{
Xian-feng Man, ${ }^{1,2}$ Jian Liu, ${ }^{1}$ Zhen Luo, ${ }^{2}$ Bai-zhan Xia ${ }^{1, a)}$ \\ ${ }^{1}$ State Key Laboratory of Advanced Design and Manufacturing for Vehicle Body, Hunan University, \\ Changsha 410082, China \\ ${ }^{2}$ School of Electrical, Mechanical and Mechatronic Systems, University of Technology Sydney, \\ NSW 2007, Australia
}

In this letter, we present a class of three-dimensional (3D) labyrinthine acoustic metamaterials with self-similar fractal technique, which can produce multiple frequency-band sound insulation in deep-subwavelength scale. By simultaneously exploiting the multi-frequency bandgaps and the low-frequency characteristics, the Hilbert cubes are explored to design the 3D Hilbert fractal acoustic metamaterials (HFAMs). The multiple-band features of the HFAMs are examined by the finite element method and the effective medium theory, in which the negative bulk modulus and the mass density are responsible for the formation of the multi-bandgaps. These multi-frequency properties are induced by the Fabry-Perot multi-resonance of 3D HFAMs, which possess an ultra-high refractive index. Hence, the multi-band sound insulations of 3D HFAMs with the negative effective property are achieved below $500 \mathrm{~Hz}$. These properties of the designed 3D HFAMs provide an effective way for acoustic metamaterials to achieve multi-band filtering and noise attenuation in the low-frequency regime.

a) Electronic mail: xiabz2013@hnu.edu.cn 
Low-frequency noise attenuation or insulation has always been a challenging issue and received widespread attention in both material science and mechanical engineering due to its high penetrating power. ${ }^{1}$ Given the mass density law, the acoustic metamaterials possessing unique characteristics provide a good strategy in low-frequency sound attenuation without requiring excessive heavy bulk. $^{2,3}$

Acoustic metamaterials are artificial sub-wavelength structures with unconventional dynamic properties and remarkable functionalities, such as phononic bandgap, ${ }^{4,5}$ acoustic focusing ${ }^{6-8}$ acoustic cloaking, ${ }^{9-11}$ sound tunneling, ${ }^{11,12}$ etc. One of the most impressive features is the sound forbidden-band ability with Bragg-scattering and/or local resonance to achieve low-frequency sound attenuation. ${ }^{5,13}$ Additionally, sound absorber and attenuator possessing negative dynamic effective parameters, ${ }^{14-16}$ like including membrane-type, ${ }^{17,18}$ Helmholtz resonators, ${ }^{19,20}$ and labyrinthine structures, ${ }^{21-25}$ always play a prominent role at the low-frequency sound propagation. However, most acoustic metamaterials have only one narrow-band since the resonator in a specific configuration usually possesses one resonant mode. ${ }^{20}$ Furthermore, to certain acoustic systems requiring the multiple bandgaps, only a minimal number of bandgaps may not be suitable for some device applications. Therefore, to design multiple bandgaps at desired frequency range, an added multi-resonator, ${ }^{13,26}$ stacked/multilayer structure, ${ }^{17,27}$ parallel $^{28}$ or multiple configurations ${ }^{29}$ are necessary, while much spaces are occupied and the system complexities are increased. These restrictions have seriously hindered their potential applications. Beyond that, when these attributes, including multiple bandgaps, subwavelength characteristic, compact size and lightweight performance, are simultaneously desirable in engineering practice, the conventional acoustic metamaterials maybe become inefficient.

Inspired by the fractal design in electromagnetic waves, ${ }^{30,31}$ phononic crystals ${ }^{32}$ and acoustic 
metamaterials with hierarchy and/or self-similar fractal recently have been employed as an important strategy to explore improved acoustic properties. ${ }^{33-39}$ For instance, by using the multi-bands characteristic of fractal, a broadband acoustic metamaterial absorber with Hilbert-shaped geometry was designed to attenuate the low-frequency sound. ${ }^{36}$ Acoustic attenuators and reflectors with the self-similar fractal were further obtained for subwavelength multi-band sound blocking. ${ }^{33,37,39}$ Newly, based on stretched 2D Hilbert fractal, multi-frequency noises caused by a transformer was reduced. ${ }^{35}$ Moreover, the path length of sound propagation in labyrinthine structures also determines the performance of sound attenuation, especially at low-frequencies. ${ }^{25}$ These progresses indicate that the desired multiple and subwavelength characteristics could be achieved by rationally introducing the fractal architectures. Beyond that, the current labyrinthine structures with the self-similar fractal were dominated by 2D cases, and designing 3D fractal labyrinthine configurations have been rarely explored. In this letter, we present a class of subwavelength 3D Hilbert-shaped metamaterials aimed to explore the acoustic property, in which the multi-band bandgaps and the low-frequency characteristics are obtained simultaneously. Furthermore, multi-band negative effective bulk modulus and mass density are generated to explain multi-band sound attenuation, in which the Fabry-Perot resonances occur. The designed 3D novel features beyond capabilities of 2D bio-inspired hierarchical structures can be applied to sound insulation of multiple frequency-bands in deep-subwavelength scales. $^{33,34}$

Figure. 1(a)-(c) show schematic plots of the 3D HFAMs unit cells, which are shaped by Hilbert cubes based on a software openSCAD. Note that the difference between Hilbert cubes and proposed HFAMs structures is that the ends of the segment are connected with the outside, forming a 3D Hilbert cubes channel with width $d$, as are shown in Fig. 1(d)-(f). At different level $n$, the cube is now partitioned into $8^{n}$ sub-cubes and the path consists of $\left(8^{n}-1\right) a_{0}$ segments of length $1 / 2^{n} a_{0}$, leading to a 
length of $\left[\left(8^{n}-1\right) / 2^{n}\right] a_{0} . a_{0}$ is the edge length of Hilbert cubes. Hence, the wave propagation length of Hilbert cube channel is elongated by $L_{\text {eff }}=4^{n} a_{0}(n=1,2,3)$, which is much longer than structures formed by $2 \mathrm{D}$ Hilbert curve $\left(2^{n} a_{0}\right)^{33,34}$ and Wunderlich curve $\left(3^{n} a_{0}\right) .{ }^{37}$

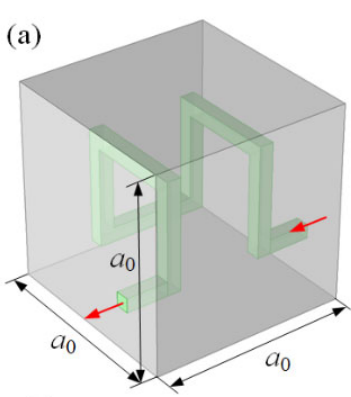

(d)

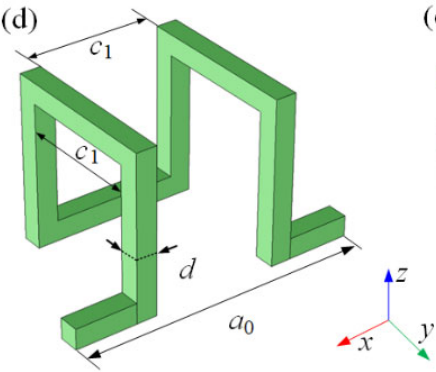

(b)

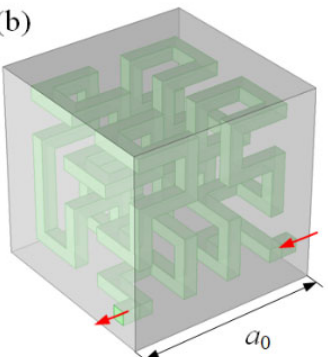

(e)

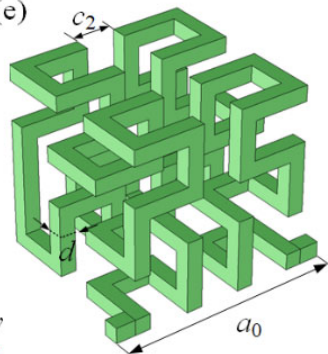

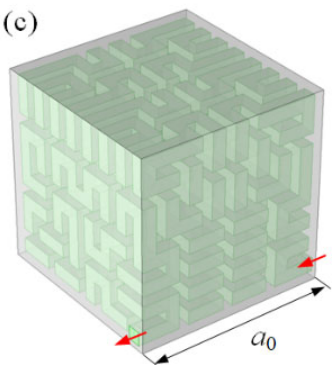

(f)

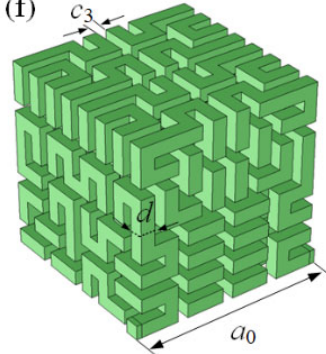

FIG. 1. The unit cell models of 3D HFAMs. (a)-(c): unit cells of first three-orders HFAMs. Gray area of Hilbert cubes is epoxy. (d)-(f): the independent Hilbert cubes channels with width $d=5 \mathrm{~mm}$. The unit cells are arranged in a simple cubic (SC) lattice. The parameters of HFAMs are follows: the edge length of Hilbert cubes $a_{0}=70 \mathrm{~mm}$, the lattice constant $a=75 \mathrm{~mm}$, the spacings of beams inside Hilbert cells are $c_{1}=30 \mathrm{~mm}, c_{2}=12.5 \mathrm{~mm}, c_{3}=3.75 \mathrm{~mm}$, respectively. The red arrows represent the entrance and exit of sound waves.

In 3D HFAMs, the acoustic wave propagates along the Hilbert paths, whose effective lengths are several times longer than the straight channel extending from the left to right port of Hilbert cube, resulting in the propagation is delayed. Hence, the proposed HFAMs have an equivalent high refractive index and ultra-slow speed compared to $2 \mathrm{D}$ cases. Note that as the order increases, the interiors of Hilbert cubes are filled with air channels, showing its lightweight feature. As mentioned above, the 3D HFAMs not only possess the features of miniaturization, lightweight and high refractive index, but also realize the multi-bands and the low-frequency characteristics simultaneously.

Considering that the solid material's impedance is much larger than that of air, the solid walls can be regarded as the sound-hard boundary condition. ${ }^{23}$ In air domain, the Helmholtz equation of 
acoustic wave propagation can be written as

$$
\nabla\left(-\frac{1}{\rho_{0}} \nabla p\right)+\frac{1}{\rho_{0} c_{0}^{2}} \omega^{2} p=0
$$

Where $p$ is the sound pressure, and $\omega$ is the angular frequency, and $\rho_{0}=1.25 \mathrm{~kg} / \mathrm{m}^{3}$ and $c_{0}=343 \mathrm{~m} / \mathrm{s}$ are the mass density and speed of sound, respectively. To capture the periodic feature of HFAMs, Floquet-Bloch's periodic conditions are applied at the boundaries of the unit cells. ${ }^{33}$ To investigate the band structure of HFAMs, acoustic dispersions are performed by eigenfrequency analysis using the COMSOL Multiphysics. We then solve the wave equation by scanning the wave vectors $\boldsymbol{k}$ in the first irreducible Brillouin zone (IBZ) as shown in the inset of Fig. 2(a).

Figure. 2(a)-(c) shows the band structures of HFAMs in terms of subwavelength frequency range. For the first-order HFAM as shown in Fig. 2(a), it can be observed that only two small full bandgaps $[629.63 \mathrm{~Hz}, 640.4 \mathrm{~Hz}]$ and $[1200.3 \mathrm{~Hz}, 1258.8 \mathrm{~Hz}]$, respectively. For the second-order HFAM, there are more bandgaps appearing, i.e., $[164.66 \mathrm{~Hz}, 195.89 \mathrm{~Hz}],[325.28,329.04 \mathrm{~Hz}],[492.4 \mathrm{~Hz}$, $501.13 \mathrm{~Hz}],[652.13 \mathrm{~Hz}, 659.13 \mathrm{~Hz}],[976.42 \mathrm{~Hz}, 986.98 \mathrm{~Hz}]$. For the third-order HFAM, more bandgaps are produced than that of the first two ones in lower frequency range $[0,500 \mathrm{~Hz}]$, i.e., $[44.36 \mathrm{~Hz}, \quad 72.138 \mathrm{~Hz}], \quad[133.09 \mathrm{~Hz}, 150.12 \mathrm{~Hz}], \quad[221.8 \mathrm{~Hz}, \quad 233.21 \mathrm{~Hz}], \quad[310.49 \mathrm{~Hz}, 319.22 \mathrm{~Hz}]$, $[399.2 \mathrm{~Hz}, 405.72 \mathrm{~Hz}],[487.92 \mathrm{~Hz}, 492.41 \mathrm{~Hz}]$. We can find that: firstly, as the fractal order increases, the higher-order fractals have more bandgaps, and the frequency-bands move to the lower-frequencies range. Secondly, their relative bandwidths of the first bandgap are abundantly broadened from $1.7 \%$ to $8.7 \%$, and then to $23.8 \%$, respectively. Thirdly, for the third-order HFAMs, the lower-bound of the first bandgap frequency has an ultra-low-frequency, and its normalized frequency is $f a / c_{0}=0.0097$, which is a deep-subwavelength scale. The lowest bandgap frequency is nearly one-order of magnitude lower than that of the first one. In addition, the transmission characteristics of HFAMs with 5- and 10-unit cells are evaluated, as shown in the right panels of Fig. 
2(a)-(c). It is clearly seen that sound waves are strongly attenuated in the frequency bands along the

ГX direction, and their locations match well with the bandgaps.

It is clearly noted that the bands along $\mathbf{R M}$ and $\mathbf{X M}$ directions are completely flat, especially the second and third-order HFAMs (Fig. 2(b)-(c)). These flat-bands are located in correspondence of the Fabry-Perot resonance, ${ }^{8,40} f_{\mathrm{p}} \approx n c 0 / 2 L_{\text {eff, }}$ with $n$ is a positive integer. These flat-bands indicate that the existence of localized modes, that also have zero group velocity, which also present a localized pattern. In addition, as shown in Fig. 2(a)-(c), the red dotted lines represent their phase velocities of the first dispersion band of HFAMs at high symmetry point $\Gamma$ are $267.47 \mathrm{~m} / \mathrm{s}, 213 \mathrm{~m} / \mathrm{s}$ and $95.92 \mathrm{~m} / \mathrm{s}$, respectively. In particular, the two branches of each band can almost coincide with each other at the point $\boldsymbol{\Gamma}$ along the $\boldsymbol{\Gamma X}$ and $\boldsymbol{\Gamma \mathbf { M }}$ directions. ${ }^{21,23}$ This indicates that the proposed 3D HFAMs can be considered as an isotropic structure in considered frequency range.

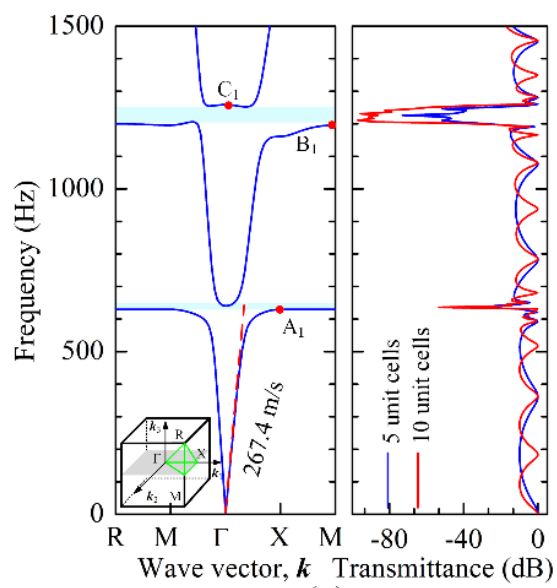

(a)

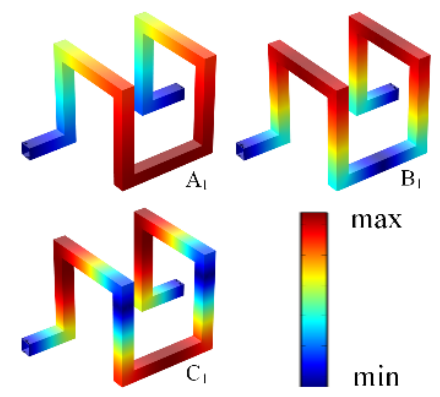

(d)

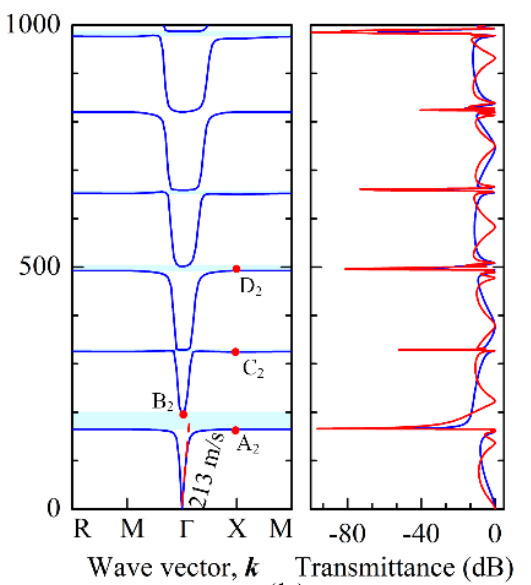

(b)

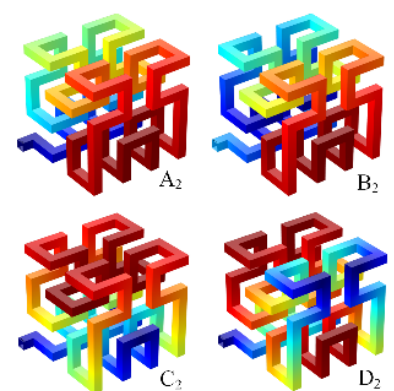

(e)

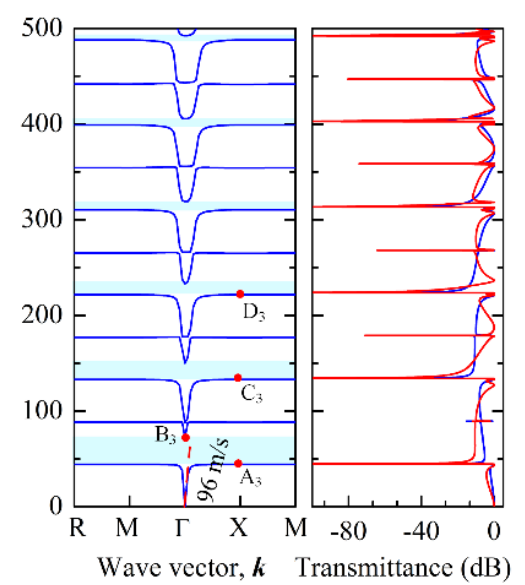

(c)
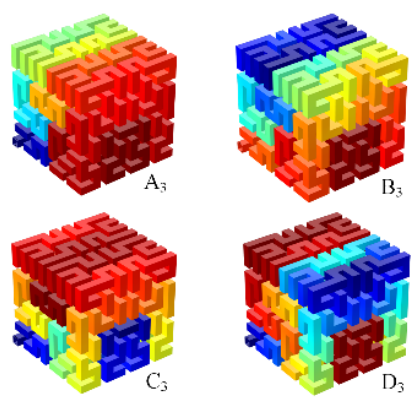

(f)

FIG. 2 (a)-(c) Band structures and transmittances of 3D HFAMs. The inset shows the first IBZ in the reciprocal space for SC lattices. Full bandgaps are highlighted in light-blue. (d)-(f) Pressure distributions around bandgaps boundary $\mathrm{A}_{i}-\mathrm{D}_{i}(i=1,2,3)$ for $3 \mathrm{D}$ Hilbert cells. 
To further gain a deeper understanding, we plot the acoustic modes of HFAMs at the points $\boldsymbol{\Gamma}$, M, and K. Here, to observe the sound pressure distributions inside 3D HFAMs, Hilbert channels cells (Fig. 1(d)-(f)) are selected instead of HFAMs (Fig. 1(a)-(c)). The pressure profiles of Hilbert channels are superimposed in Fig. 2(d)-(f), which are similar to artificial monopole, dipole and multipole resonances patterns. For example, the low-bounds of first bandgap $\mathrm{A}_{i}$ are similar to a monopole, in which the pressure is concentrated in the central part of Hilbert channel, equally radiating along two propagation directions. ${ }^{22,24}$ Thus, the monopole and multipole resonances in folded channels originate from the Fabry-Perot resonances. ${ }^{37}$ Those resonance features can induce negative effective properties, which can generate a high reflection, resulting in the attenuation of

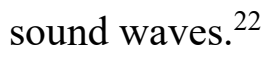

The use of effective medium approach can be used for extracting extreme properties under long-wavelength assumption. The transfer-matrix method (TMM) is employed to relate the normal sound velocities and sound pressures on both sides of a mono-layer cell extending from $x=0$ to $x=d$ as in Fig. 3(a),

$$
\left[\begin{array}{l}
P_{l} \\
V_{l}
\end{array}\right]_{x=0}=\boldsymbol{T}_{e}\left[\begin{array}{l}
P_{r} \\
V_{r}
\end{array}\right]_{x=d}=\left[\begin{array}{ll}
T_{11} & T_{12} \\
T_{21} & T_{22}
\end{array}\right]\left[\begin{array}{l}
P_{r} \\
V_{r}
\end{array}\right]_{x=d}
$$

Where $P_{i}$ and $V_{i}(i=l, r)$ are the complex pressures and normal sound velocities on the left and right sides of unit cell.

$$
\boldsymbol{T}_{e}=\left[\begin{array}{ll}
\frac{P_{r} V_{r}+P_{l} V_{l}}{P_{r} V_{r}+P_{r} V_{r}} & \frac{P_{l}^{2}-P_{r}^{2}}{P_{l} V_{r}+P_{r} V_{l}} \\
\frac{V_{l}^{2}-V_{r}^{2}}{P_{l} V_{r}+P_{r} V_{l}} & \frac{P_{r} V_{r}+P_{l} V_{l}}{P_{r} V_{r}+P_{r} V_{r}}
\end{array}\right]=\left[\begin{array}{cc}
\cos \left(k_{\mathrm{eff}} d\right) & j Z_{\mathrm{eff}} \sin \left(k_{\mathrm{eff}} d\right) \\
j \sin \left(k_{\mathrm{eff}} d\right) / Z_{\mathrm{eff}} & \cos \left(k_{\mathrm{eff}} d\right)
\end{array}\right]
$$

Where $k_{\text {eff }}$ is a complex wave number, and the complex characteristic impedance can be calculated from $Z_{\text {eff }}=\rho_{\text {eff }} \omega / k_{\text {eff. }}$ With the dispersion relation and Eq. (3), the effective mass density $\rho_{\text {eff }}$ and effective bulk modulus $B_{\text {eff }}$ normalized to the background air are given by 


$$
\rho_{\text {eff }}=\frac{j k_{x} \sin k_{x} d}{\omega T_{21} \rho_{0}}, \quad B_{\text {eff }}=\frac{\omega^{2}}{3 k_{\text {eff }} / \rho_{\text {eff }}} / \rho_{0} c_{0}^{2}
$$

To verify the correctness of the TMM, the $S$-parameter retrieval method have been also used for characterizing 3D HFAMs. ${ }^{41}$ By using the complex transmission and reflection coefficients of a monolayer cell as shown in Fig. 3(b), $B_{\text {eff }}$ and $\rho$ eff can also be obtained.
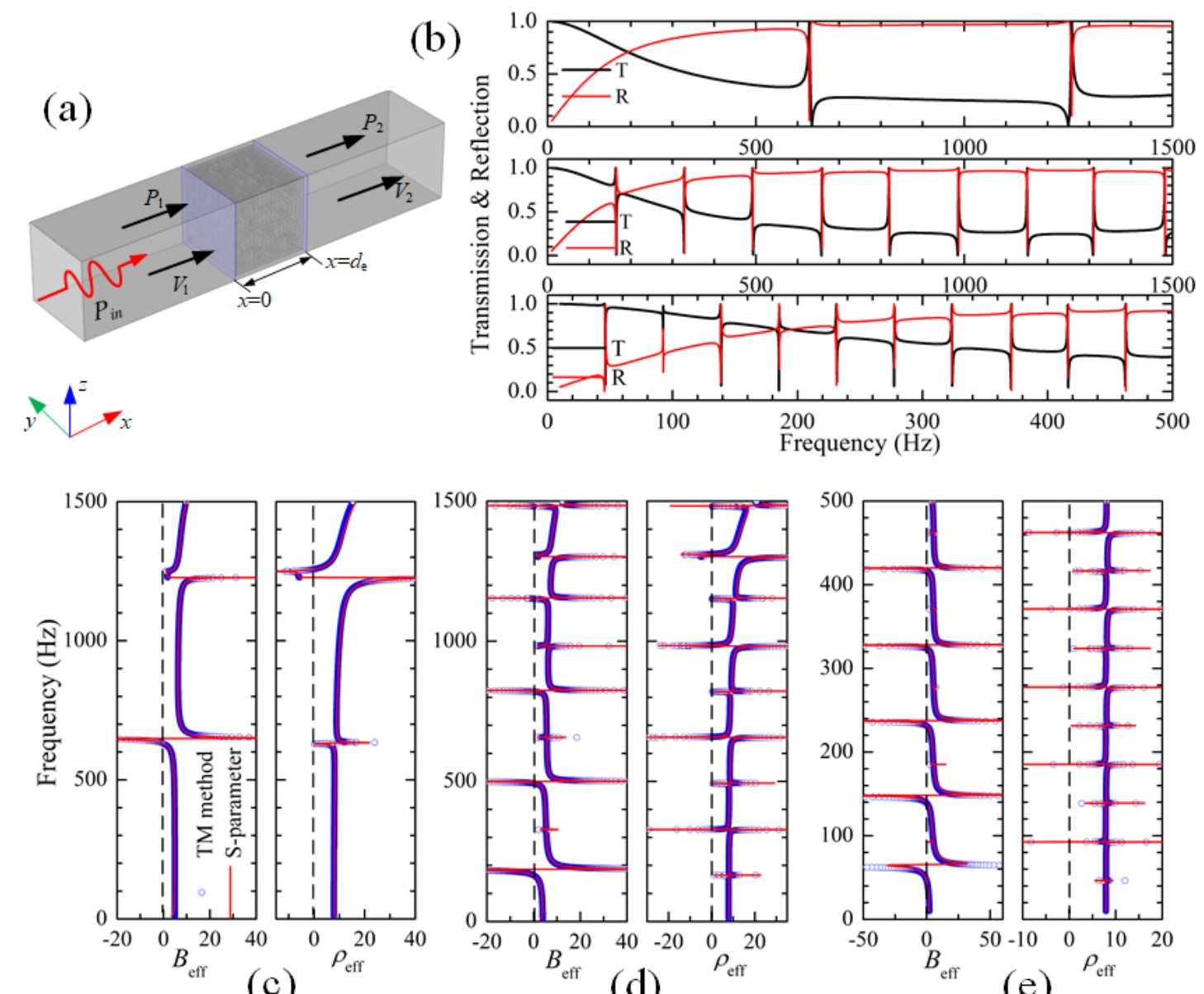

(d)

(e)

FIG. 3. The transmission characteristics and effective parameters of 3D HFAMs. (a) Schematic diagram of the TMM. (b) The transmission (black solid lines) and reflection (red solid lines) of unit cells of HFAMs. (c)-(e) show the $B$ eff and $\rho_{\text {eff }}$ of HFAMs based on TMM (blue circle) and $S$-parameter method (red solid line), respectively.

Figure. 3(c)-(e) represent $B_{\text {eff }}$ and $\rho_{\text {eff }}$ of the 3D HFAMs predicted by the TMM and $S$-parameter method, in which their results are consistent. It is clearly observed that Fig. 3(c)-(e) exhibit very rich negative behaviors for each $B_{\text {eff }}$ and $\rho_{\text {eff }}$ even at such low-frequencies, and correspond to the transmission resonant characteristics in Fig. 3(b). For the first-order HFAMs in Fig. 3(c), there is a negative $B_{\text {eff }}$ and $\rho_{\text {eff }}$ in one narrow frequency-band around $639 \mathrm{~Hz}$ and $1250 \mathrm{~Hz}$, respectively. For the 
second-order HFAMs in Fig. 3(d), $B_{\text {eff }}$ first turns to be negative from $164.8 \mathrm{~Hz}$ to $186.1 \mathrm{~Hz}$, then in other narrow bands around $492 \mathrm{~Hz}, 820.3 \mathrm{~Hz}, 1151.8 \mathrm{~Hz}$, and $1482.3 \mathrm{~Hz}$; $\rho_{\text {eff }}$ become negative around $328 \mathrm{~Hz}, 657.1 \mathrm{~Hz}, 983 \mathrm{~Hz}, 1301.8 \mathrm{~Hz}$, respectively. For the third-order 3D HFAMs in Fig. 3(e), it is seen that $B_{\text {eff }}$ produces negative values in four narrow bands around $46.3 \mathrm{~Hz}, 138.9 \mathrm{~Hz}, 231.4 \mathrm{~Hz}$, and $323.8 \mathrm{~Hz}$; the negative $\rho_{\text {eff }}$ arises in five narrow bands around $92.6 \mathrm{~Hz}, 185.3 \mathrm{~Hz}, 277.5 \mathrm{~Hz}, 371 \mathrm{~Hz}$ and $462.4 \mathrm{~Hz}$, respectively.

Note that as the order increases, the number of negative frequency-bands of $B_{\text {eff }}$ and $\rho_{\text {eff }}$ increases gradually, and the negative bands move to the lower-frequencies. Moreover, the negative $B_{\text {eff }}$ and $\rho_{\text {eff }}$ take place all around the resonant frequencies. It is also noticed that negative regions of $B_{\text {eff }}$ and $\rho_{\text {eff }}$ coincide with the bandgaps range. In particular, to the third-order HFAMs, it appears the negative frequency-bands of $B_{\text {eff }}$ and $\rho_{\text {eff }}$ in the ultra-low-frequency range blew $100 \mathrm{~Hz}$.

We perform the sound transmission loss (STL) of 3D HFAMs with one monolayer cell to confirm our analysis, as shown in Fig. 4(a)-(c). The STLs are calculated by average sound pressure in the cross-section of output of a waveguide, which is show in Fig. 3(a). For the first two-order HFAMs, there are two and nine transmission dips at the frequencies range [0,1500Hz], respectively. For comparison, STL (the blue circle) of a rigid cube with the same size by calculating the average sound pressure of output is illustrated in Fig. 4(a), in which the rigid cube does not produce sound attenuation. For the second-order HFAM, the structure size is almost $1 / 10$ wavelength at resonant frequency around $493.5 \mathrm{~Hz}$ with the wavelength of $700 \mathrm{~mm}$. Furthermore, Fig. 4(c) displays that the third-order HFAM can produce more frequency-bands of sound attenuation because it possesses much higher refractive index. Therefore, the proposed higher-order HFAMs with deep-subwavelength scales can produce low-frequency sound attenuation at multiple frequency-bands than $2 \mathrm{D}$ cases $^{34}$ and membranes-type insulator. ${ }^{42,43}$ To analyze the reason of the 
high sound attenuation, the right panel of Fig. 4 shows 3D sound pressure level (SPL) distributions and velocity profiles of 3D Hilbert channels at different resonant frequencies. It is clearly seen that most of the acoustic energy is concentrated inside Hilbert channels, leading to the sound attenuation through the monolayer structure. Additionally, Fig. 4(d), (f) and (j) show the SPL distributions of local velocity profiles of air particles of HFAM at the first attenuation frequencies of $634.5 \mathrm{~Hz}$, $165.1 \mathrm{~Hz}$, and $46.3 \mathrm{~Hz}$, respectively. A clear inverse velocities flow is observed in the input and output ports of HFAM's SPL, which originates from negative bulk modulus induced by the Fabry-Perot fundamental resonance. This similar situation occurs at the third frequency of $493.5 \mathrm{~Hz}$ and $133.9 \mathrm{~Hz}$, shown in Fig. 4(h) and (1). In addition, as shown in Fig. 4(e), (g) and (k), while the cases are different for the second frequencies of HFAM at $1250 \mathrm{~Hz}, 327.7 \mathrm{~Hz}$ and $89.1 \mathrm{~Hz}$, respectively. The air velocities flow in the form of circular patterns, which originate from negative mass densities.
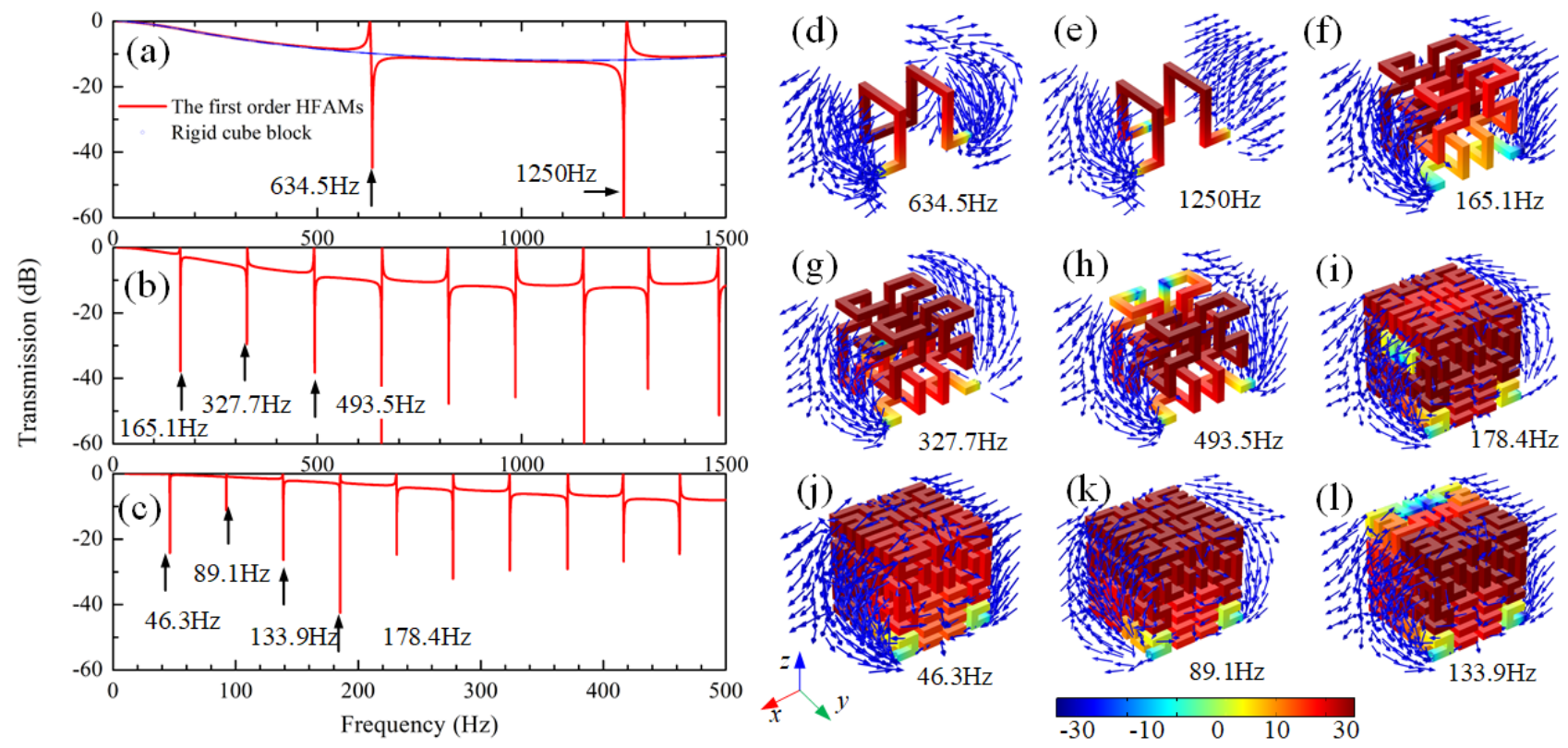

FIG. 4. (a)-(c) STL of a monolayer cell of 3D HFAMs (red solid lines) and the rigid cube block with same size (blue circle). (d)-(1) 3D SPL distributions and velocity profiles of Hilbert channels at different resonant frequencies. The blue arrows show the direction of the air velocity. The length size of the arrows is normalized into better observations.

In summary, we have proposed and investigated a class of 3D HFAMs inspired by Hilbert-shaped fractal architectures, which can be as sound insulations with multi-bands and 
lightweight characteristics in deep-subwavelength scales. We find that the number of bandgaps increase and the bandgaps gradually shift to low-frequencies regime as the order of fractal increases. Remarkably, the multi-bands characteristic of 3D HFAMs are attributed to the Fabry-Perot resonance, which induce the multi-bands negative effective bulk modulus and mass density, resulting in acoustic wave suppression. Therefore, these properties exhibit that proposed 3D HFAMs with the deep-subwavelength scales can produce sound insulations of multiple frequency-bands below $500 \mathrm{~Hz}$, while the airflows are not restricted.

\section{Acknowledgments}

This work was supported by the Joint Fund of Ministry of Education for Equipment Pre-Research (6141A02033216), and Australian Research Council (ARC) - Discovery Projects (160102491), as well as the China Scholarship Council (201706130093) and Hunan Provincial Innovation Foundation for Postgraduate (CX2016B080). X.F. M would like to thank Dr. A.O. Krushynska for her discussions, and D. Melcer for help on the models.

\section{Reference}

${ }^{1}$ J. S. Bolton, N.-M. Shiau, Y. J. Kang, J. Sound. Vib. 191, 31 (1996).

${ }^{2}$ Z. Yang, H. M. Dai, N. H. Chan, G. C. Ma, and P. Sheng, Appl. Phys. Lett. 96, 041906 (2010).

${ }^{3}$ Z. Yang, J. Mei, M. Yang, N. H. Chan, and P. Sheng, Phys. Rev. Lett. 101, 204301 (2008).

${ }^{4}$ M. Badreddine Assouar, M. Senesi, M. Oudich, M. Ruzzene, and Z. Hou, Appl. Phys. Lett. 101, 173505 (2012).

${ }^{5}$ Z. Liu, X. Zhang, Y. Mao, Y. Y. Zhu, Z. Yang, C. T. Chan, and P. Sheng, Science 289, 3 (2000).

${ }^{6}$ L. Zigoneanu, B. I. Popa, and S. A. Cummer, Phys. Rev. B 84 (2011).

${ }^{7}$ B. Yuan, Y. Cheng, and X. Liu, Appl. Phys. Express 8, 027301 (2015).

${ }^{8}$ Y. Li, B. Liang, X. Tao, X. F. Zhu, X. Y. Zou, and J. C. Cheng, Appl. Phys. Lett. 101, 233508 (2012).

${ }^{9}$ S. Zhang, C. Xia, and N. Fang, Phys. Rev. Lett. 106 (2011).

${ }^{10}$ L. Y. Zheng, Y. Wu, X. Ni, Z. G. Chen, M. H. Lu, and Y. F. Chen, Appl. Phys. Lett. 104, 161904 (2014).

${ }^{11}$ X. F. Fu, G. Y. Li, M. H. Lu, G. Lu, and X. Huang, Appl. Phys. Lett. 111, 251904 (2017).

${ }^{12}$ S. Yang, J. H. Page, Z. Liu, M. L. Cowan, C. T. Chan, and P. Sheng, Phys. Rev. Lett. 88 (2002).

${ }^{13}$ L. Jing, J. H. Wu, D. Guan, and N. Gao, J. Appl. Phys. 116, 103514 (2014).

${ }^{14}$ H. Chen, H. Li, S. Zhai, C. Ding, J. Li, C. Luo, and X. Zhao, J. Appl. Phys. 119, 204902 (2016).

${ }^{15}$ P. Sheng, G. C. Ma, Science Advances 2 (2016).

${ }^{16}$ S. A. Cummer, J. Christensen, and A. Alù, Nat. Rev. Mater. 1, 16001 (2016). 
${ }^{17}$ L. Fan, Z. Chen, S. Y. Zhang, J. Ding, X. J. Li, and H. Zhang, Appl. Phys. Lett. 106, 151908 (2015).

${ }^{18}$ N. Sui, X. Yan, T. Y. Huang, J. Xu, F. G. Yuan, and Y. Jing, Appl. Phys. Lett. 106, 171905 (2015).

${ }^{19}$ S. Cho, B. Kim, D. Min, and J. Park, J. Appl. Phys. 118, 163103 (2015).

${ }^{20}$ N. Fang, D. Xi, J. Xu, M. Ambati, W. Srituravanich, C. Sun, and X. Zhang, Nat. Mater. 5, 452 (2006).

${ }^{21}$ Z. X. Liang and J. Li, Phys. Rev. Lett. 108, 114301 (2012).

${ }^{22}$ Y. Cheng, C. Zhou, B. G. Yuan, D. J. Wu, Q. Wei, and X. J. Liu, Nat. Mater. 14, 1013 (2015).

${ }^{23}$ T. Frenzel, D. B. Jan, T. Bückmann, R. Schittny, M. Kadic, and M. Wegener, Appl. Phys. Lett. 103, 061907 (2013).

${ }^{24}$ A. O. Krushynska, F. Bosia, M. Miniaci, and N. M. Pugno, New. J. Phys. 19, 105001 (2017).

${ }^{25}$ Z. Chen, L. Fan, S.Y. Zhang, H. Zhang, X. J. Li, and J. Ding, Appl. Phys. Express 8, 107301 (2015).

${ }^{26}$ G. L. Huang and C. T. Sun, ASME J. Vib. Acoust. 132, 031003 (2010).

${ }^{27}$ L. Hao, M. Men, Y. Zuo, X. Yan, P. Zhang, and Z. Chen, J. Phys. D: Appl. Phys. 51, 385104 (2018).

${ }^{28}$ Y. Cheng, J. Y. Xu, and X. J. Liu, Appl. Phys. Lett. 92, 051913 (2008).

${ }^{29}$ R. Zhu, G. L. Huang, and G. K. Hu, ASME J. Vib. Acoust. 134, 031006 (2012).

${ }^{30}$ W. Wen, L. Zhou, J. Li, W. Ge, C. T. Chan, and P. Sheng, Phys. Rev. Lett. 89, 223901 (2002).

${ }^{31}$ B. Hou, H. Xie, W. Wen, and P. Sheng, Phys. Rev. B 77 (2008).

${ }^{32}$ Y. Y. Chen and L. F. Wang, Extreme Mech. Lett. 5, 18 (2015).

${ }^{33}$ X. F. Man, T. T. Liu, B. Z. Xia, Z. Luo, L. X. Xie, and J. Liu, J. Sound. Vib. 423, 322 (2018).

${ }^{34}$ X. Zhao, G. Liu, C. Zhang, D. Xia, and Z. Lu, Appl. Phys. Lett. 113, 074101 (2018).

${ }^{35}$ J. Liu, L. P. Li, B. Z. Xia, and X. F. Man, Int. J. Solids Struct. 132-133, 20 (2018).

${ }^{36}$ G. Y. Song, Q. Cheng, B. Huang, H. Y. Dong, and T. J. Cui, Appl. Phys. Lett. 109, 131901 (2016).

${ }^{37}$ A. O. Krushynska, F. Bosia, and N. M. Pugno, Acta Acust. united Ac 104, 200 (2018).

${ }^{38}$ M. Miniaci, A. Krushynska, A. S. Gliozzi, N. Kherraz, F. Bosia, and N. M. Pugno, Phys. Rev. Appl. 10 (2018).

${ }^{39}$ B. Z. Xia, L. P. Li, J. Liu, and D. J. Yu, ASME J. Vib. Acoust. 140, 011011 (2017).

${ }^{40}$ M. Molerón, M. Serra-Garcia, and C. Daraio, New. J. Phys. 18, 033003 (2016).

${ }^{41}$ V. Fokin, M. Ambati, C. Sun, and X. Zhang, Phys. Rev. B 76 (2007).

${ }^{42}$ W. W. Yu, L. Fan, R. H. Ma, H. Zhang, and S. Y. Zhang, Appl. Phys. Lett. 112, 183506 (2018).

${ }^{43}$ G. Ma, M. Yang, Z. Yang, and P. Sheng, Appl. Phys. Lett. 103, 011903 (2013). 
(a)

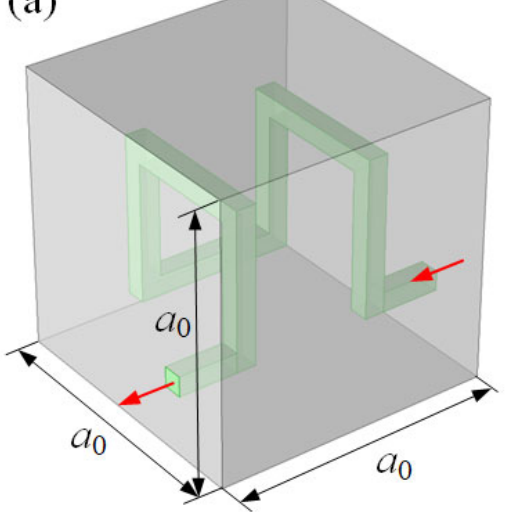

(d)

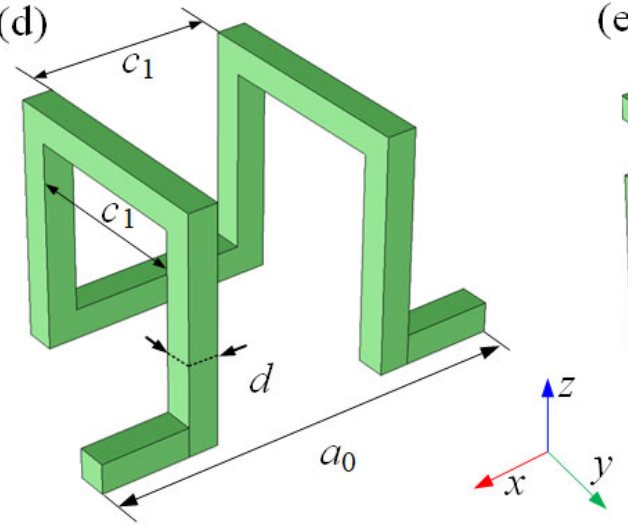

(b)

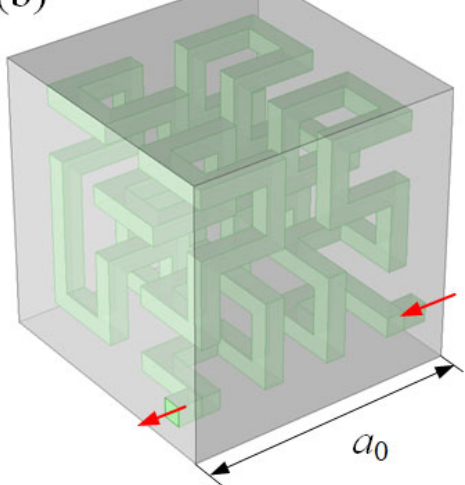

(e)

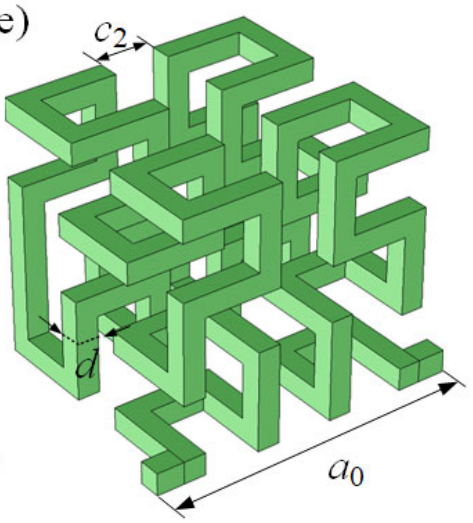

(c)

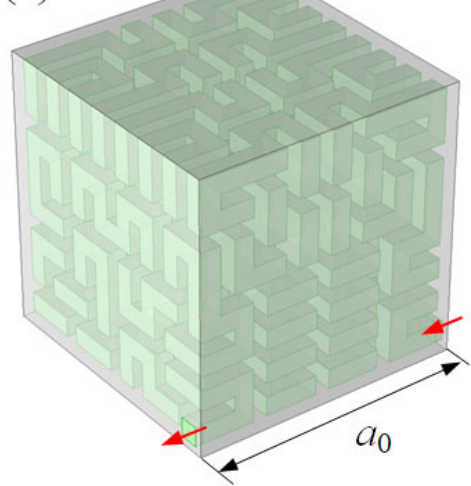

(f)

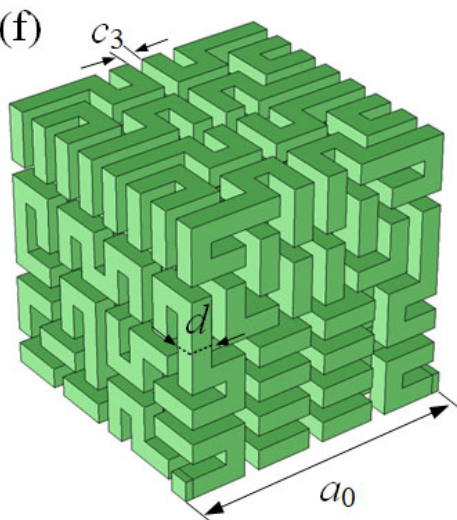




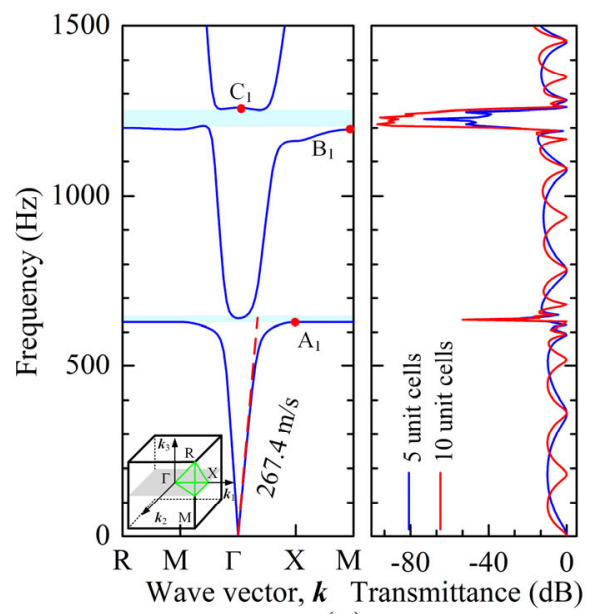

(a)

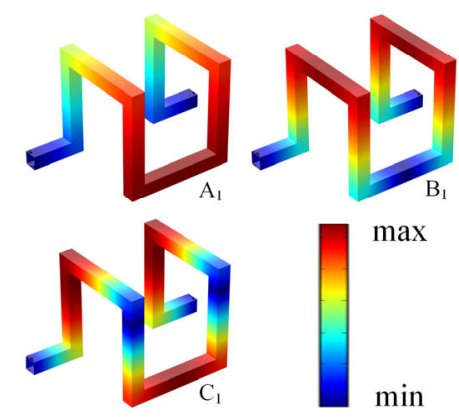

(d)

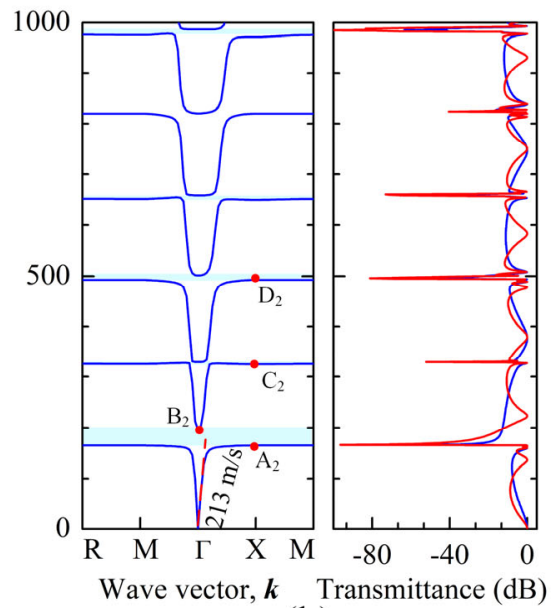

(b)

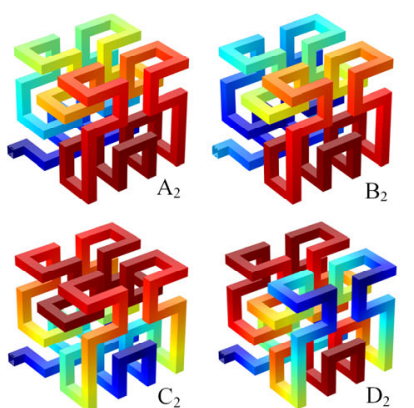

(e)

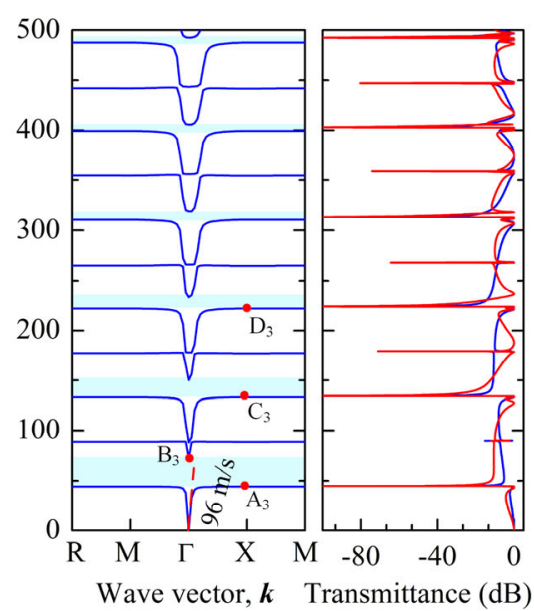

(c)

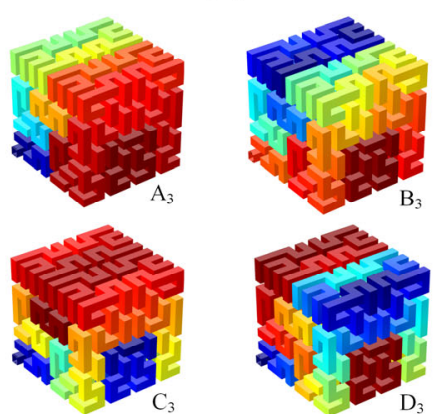

(f) 
(b)
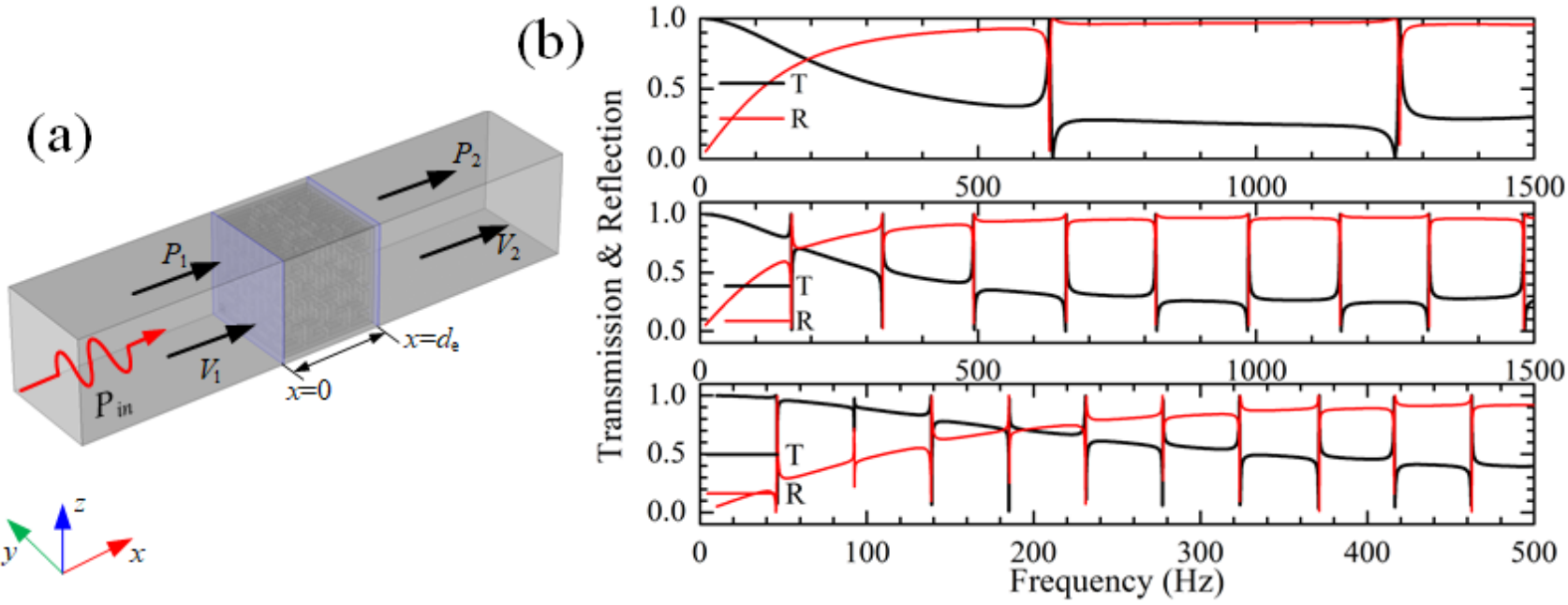

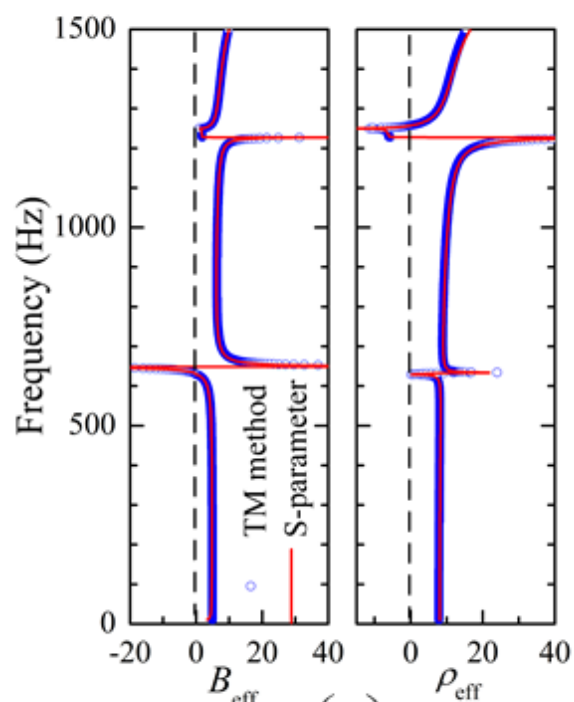

(c)

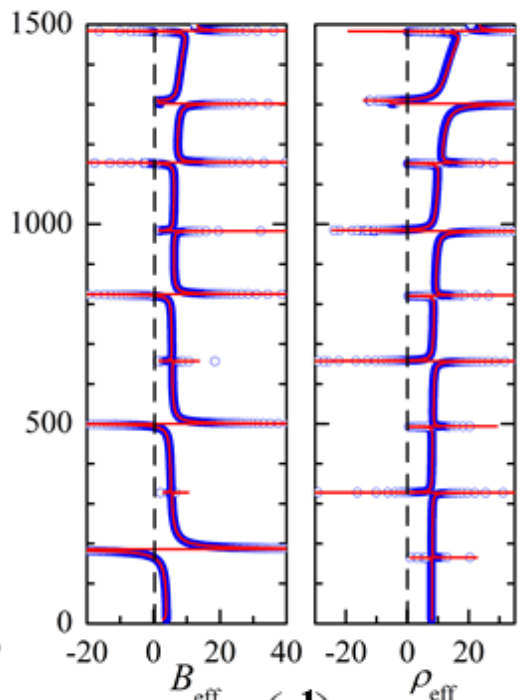

(d)

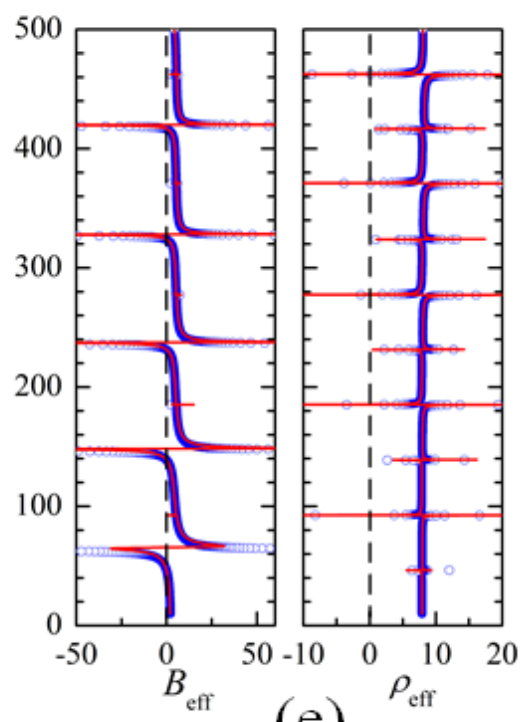

(e) 

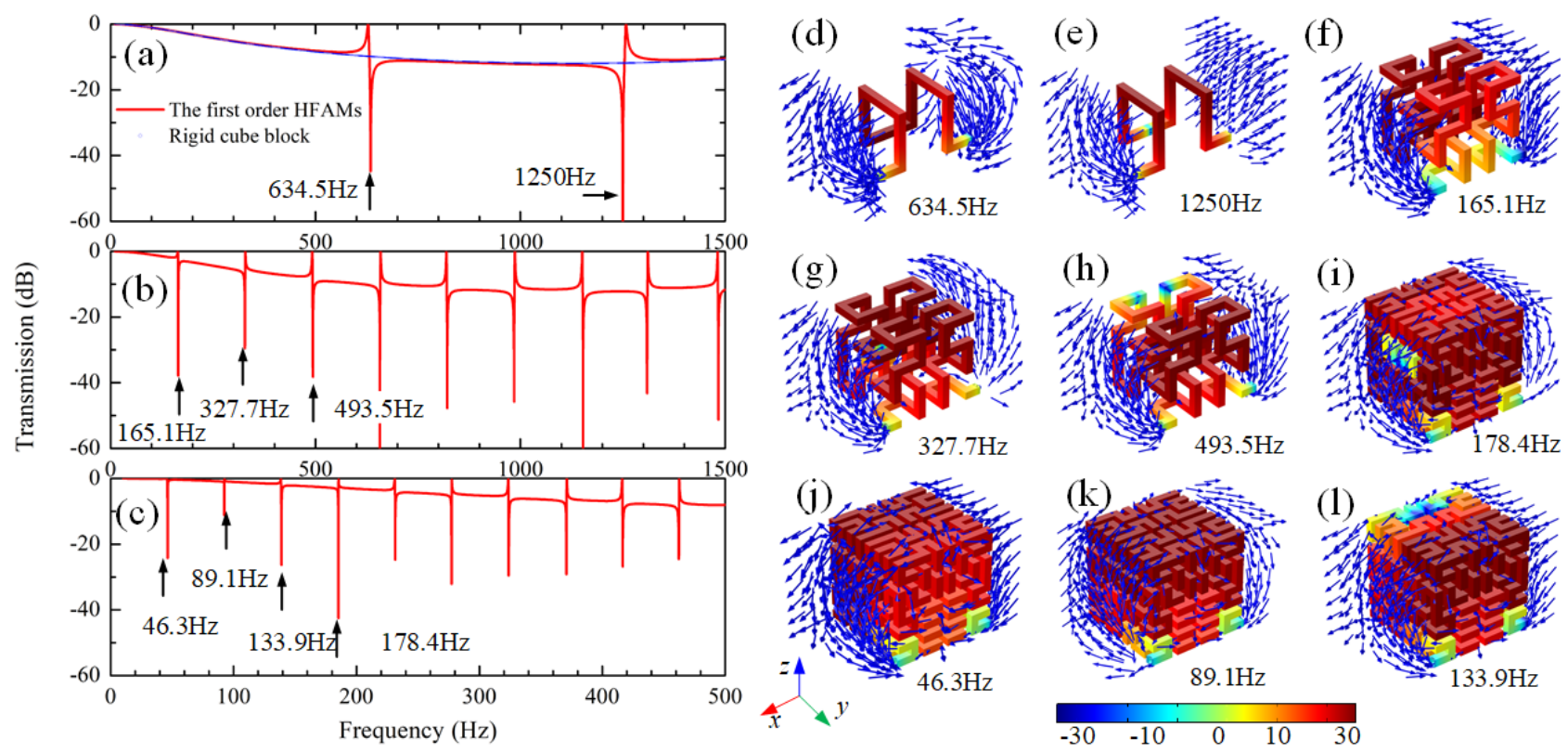

(h)
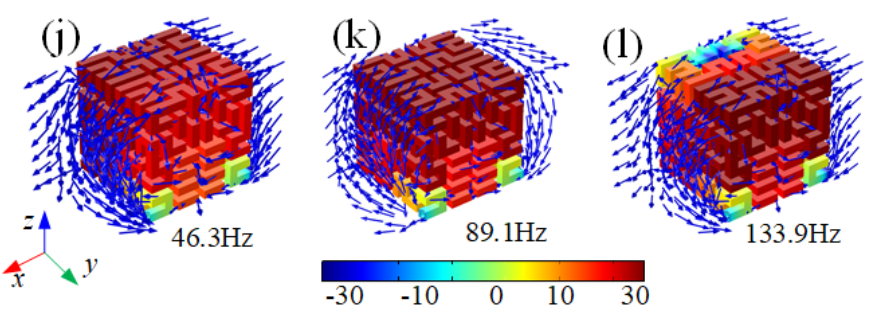